\title{
Genetic modulation of self renewal factors for induced reprogramming of somatic cells
}

\author{
Holm Zaehres ${ }^{1}$, Jeong Tae Do ${ }^{1}$, Nishant Singhal ${ }^{1}$, Natalia Tapia ${ }^{1}$, Dong Wook Han ${ }^{1}$, Hans Schöler ${ }^{1}$ \\ ${ }^{1}$ Max Planck Institute for Molecular Biomedicine, Dept. Cell and Developmental Biology, Münster, NRW 48149, Germany
}

Studies examining somatic cell nuclear transfer (SCNT) have demonstrated that the cytoplasm of an oocyte can reprogram the genome of a somatic cell to an embryonic state. Reprogramming of a somatic cell to a pluripotent state can also be achieved either by fusion with embryonic stem cells or by the ectopic expression of defined transcription factors. Pluripotent stem cells derived by this means can be differentiated in defined tissues, and serve as model systems for the study and treatment of human diseases. Using the first application of stable RNA interference in human embryonic stem cells, we showed that knockdown of Oct4 and Nanog gene expression promotes their differentiation into cells expressing trophectodermal and endodermal markers, thereby demonstrating a conserved role for these factors in the self - renewal of human embryonic stem cells (Zaehres et al., 2005). We have developed retro / lentivirus vectors for efficient transgene expression in mouse and human embryonic stem cells as well as progenies derived thereof (Zaehres and Daley, 2006). We are further developing transgenic reporter systems expressing green fluorescent protein (GFP) and other selection markers under the control of mouse and human Oct4 and Nanog promoter elements.

Currently we are applying these vector systems to explore reprogramming induced by fusion of somatic cells with mouse and human embryonic stem cells and to explore reprogramming of mouse and human somatic cells with defined transcription factors.

These technologies are instrumental in providing a detailed, molecular understanding of the genomic reprogramming process with the ultimate goal to induce pluripotent cells in vitro and in vivo by the use of defined transcription factors and small molecules.

Keywords: reprogramming, retrovirus / lentivirus expression / RNAi vectors, Oct4, Nanog

Cell Research (2008) 18:s16. doi: 10.1038/cr.2008.106; published online 4 August 2008

Correspondence: Holm Zaehres

E-mail: holm.zaehres@mpi-muenster.mpg.de

Holm Zaehres has studied Biochemistry and Molecular Biology at Hamburg Medical School. He holds a PhD in Molecular Biology from Heinrich-Heine-University Düsseldorf. After some years as a Research
Fellow at Harvard Medical School, Children's Hospital Boston, he is currently a Research Scientist at the Max-Planck-Institute for Molecular Biomedicine, M ster, NRW, Germany. His major reseach interests embrace genetic engineering of stem cells, expression vectors for gene transfer and RNA interference, molecular biology of pluripotency, and reprogramming of mouse and human cells. 